\title{
Ernährungszustand und Körperzusammensetzung bei COPD - Was wissen wir?
}

\author{
Nutritional Status and Body Composition in COPD - \\ What do we know?
}

D. Bösch

\section{Lernziele \\ $\nabla$}

Der Leser soll die Relevanz (Häufigkeit und Konsequenzen) der Störungen der Körperzusammensetzung bei COPD verstehen, einen Überblick über die Möglichkeiten der entsprechenden Diagnostik bekommen und verschiedene Messparameter in diesem Zusammenhang kennenlernen.

\section{Einleitung \\ $\nabla$}

Die COPD zählt, bei einer Prävalenz von über 13\% in der deutschen Bevölkerung (> 40 Jahren) [1] heute zweifelsohne zu den Volkskrankheiten. Es wird darüber hinaus davon ausgegangen, dass die Zahl der Erkrankten in den nächsten Jahren sogar weiter steigen wird. In den letzten Jahren haben wir einen deutlichen Wissenszuwachs bzgl. der komplexen pathogenetischen Zusammenhänge und der vielschichtigen systemischen Auswirkungen und Komplikationen verzeichnen können $[2,3]$. Wir wissen heute, dass das Ausmaß der systemischen Konsequenzen in erheblichem Maße für die Prognose und die Morbidität mitverantwortlich ist [4]. Die Berücksichtigung der systemischen Auswirkungen erhält daher in zunehmendem Maße Einzug in die tägliche Praxis und findet Ausdruck in modernen Behandlungskonzepten [5]. Die Evaluation und günstige Beeinflussung des Ernährungszustands ist eine wesentliche Komponente in der Behandlung systemischer Konsequenzen der COPD und ist Gegenstand einer Vielzahl wissenschaftlicher Arbeiten in den letzten Jahren [6]. $\bullet$ Abb. 1 zeigt eine 59-jährige Patientin mit typischem Habitus bei Vorliegen einer schwergradigen COPD und einem BMI von 16 $\mathrm{kg} / \mathrm{m}^{2}$ (pulmonale Kachexie).

Bei der Beurteilung der Körperzusammensetzung können verschiedene Modelle angewandt werden. Das einfachste Modell berücksichtigt lediglich das Körpergewicht in Relation zur Körpergröße (Einkompartment-Modell). Im klassischen Zweikompartment-Modell wird zwischen Fettmasse und fettfreier Masse (FFM) unterschieden.
Die Messung mittels Densitrometrie (hydrostatisches Wiegen im Wassertank) wird vielfach als Goldstandard angesehen. Sie ist allerdings sehr aufwendig und für die Praxis daher ungeeignet. Deutlich einfacher ist die Messung der Hautfaltendicke an definierten Körperpunkten, mit der der Fettgehalt des Körpers abgeschätzt werden kann. Abb. 2 zeigt die Messung der Trizepshautfaltendicke mittels Kaliper.

Eine heute vielfach angewandte Methode ist die Bioimpedanzanalyse (BIA). Moderne BIA-Geräte sind in der Lage die Fett-Freie-Masse (Magermasse) in Zellmasse und extrazelluläre Masse zu unterscheiden (Dreikompartment-Modell). Wie bei allen indirekten Messverfahren ist auch hier ein standardisiertes Vorgehen zu beachten, um multiple Störeinflüsse zu minimieren. Die meisten Arbeiten beziehen sich auf diese Methode, da sie nicht-invasiv ist, akzeptable Daten liefert und auch in der Praxis angewendet werden kann. Weitere Differenzierungen (MehrkompartmentModelle) sind z.B. mittels DEXA (Dual-Energy Xray Absorptiometry) möglich, werden jedoch u.a. wegen zum Teil hoher Strahlenbelastung nur für gezielte Fragestellungen in vereinzelten Studien eingesetzt.

Die COPD hat eine große epidemiologische Relevanz. Die Erfassung und Beachtung systemischer Auswirkungen erlangt zunehmendes wissenschaftliches und klinisches Interesse. Die Körperzusammensetzung kann je nach Betrachtungsweise (Einkompartment- bzw. Mehrkompartment-Modell) mit unterschiedlichen Messmethoden untersucht werden.

\section{Wie ist die Situation bzgl.}

des Ernährungszustands bei den COPD-Patienten?

$\nabla$

Aus den Daten großer Multicenter-Studien (TORCH, UPLIFT) ist zu entnehmen, dass der Body Mass Index (BMI) bei COPD-Patienten im Mittel zwischen 25 und $26 \mathrm{~kg} / \mathrm{m}^{2}$ liegt [7,8]. Der 
Tab. 1 Beurteilung des Ernährungszustandes anhand des Body-Mass-Index.

\begin{tabular}{|c|c|}
\hline \multicolumn{2}{|c|}{ WHO-Klassifikation des Body-Mass-Index } \\
\hline BMI (in $\mathrm{kg} / \mathrm{m}^{2}$ ) & Bewertung \\
\hline$<18,5$ & Untergewicht \\
\hline $18,5-25$ & Normalgewicht \\
\hline$>25-30$ & Übergewicht \\
\hline$>30-35$ & Adipositas Grad 1 \\
\hline$>35-40$ & Adipositas Grad 2 \\
\hline$>40$ & Adipositas Grad 3 \\
\hline
\end{tabular}

Mittelwert entspricht somit weitestgehend dem der durchschnittlichen erwachsenen Bevölkerung [9] und liegt (bezogen auf die Altersgruppe) im oberen Normbereich. Der Mittelwert gibt uns jedoch keine Auskunft über die Verteilung der einzelnen Werte oder den Anteil der Patienten in den Problembereichen Untergewicht und Adipositas. Während der BMI relativ gut mit einem erhöhten Fettanteil korreliert, ist die Erfassung einer Mangelernährung mittels BMI jedoch häufig unzureichend. Die Anwendung der allgemeinen Klassifikation der WHO (s. $\bullet$ Tab. 1) mit einem Normbereich von $18,5-25 \mathrm{~kg} / \mathrm{m}^{2}$ ist für COPD-Patienten meist wenig hilfreich.

Bei Patienten > 65 Jahren wird, bei Vorliegen zusätzlicher Hinweise, heute meist ein Grenzwert von $22 \mathrm{~kg} / \mathrm{m}^{2}$ als Schwelle für das Vorliegen einer Mangelernährung (Malnutrition) definiert $[10,11]$. $\mathrm{Zu}$ bedenken ist allerdings, dass sich die Aussagekraft des BMI, ebenso wie die anderer Einzelparameter zur Erfassung des Ernährungszustandes, im Alter deutlich vermindert [11]. Die Fett-Freie-Masse zeichnet sich im Vergleich durch eine deutlich höhere Sensitivität und Spezifität aus. Sie fasst das Körperkompartiment mit den stoffwechselaktiven Organen, vornehmlich der Muskulatur, zusammen und ist somit ein indirektes Maß der Muskelmasse. Da das Problem der Kachexie, über den Gewichtsverlust hinaus, durch einen übermäßigen Abbau von Muskelmasse charakterisiert ist, ist die indirekte Erfassung der Muskelmasse mittels Bestimmung der FFM ein wertvoller Parameter. Für die Beurteilung des Ernährungszustandes und der Körperzusammensetzung bestehen jedoch keine eindeutigen oder einheitliche Definitionen oder Sollwerte.

In einer niederländischen Multicenter-Studie [12] ergab sich bei ambulanten COPD-Patienten mit GOLD-Stadium II und III ein Anteil mangelernährter Patienten von 27\%. Mangelernährung (nutritional depletion) wurde definiert als BMI $<21 \mathrm{~kg}$ / $\mathrm{m}^{2}$ und/oder als fat-free mass index bzw. FFMI (FFMI: FFM in kg/[Größe in $\left.\mathrm{m}^{2}\right]$ ) $<15 \mathrm{~kg} / \mathrm{m}^{2}$ (Frauen) bzw. $<16 \mathrm{~kg} / \mathrm{m}^{2}$ (Männer). Die Prävalenz von Patienten mit BMI $>21 \mathrm{~kg} / \mathrm{m}^{2}$ und vermindertem FFMI betrug $15 \%$, die von niedrigem BMI und zusätzlich niedrigem FFMI $11 \%$ und die von niedrigem BMI und normalem FFMI 1\%. Diese Zahlen wurden durch weitere Studien bestätigt, in denen

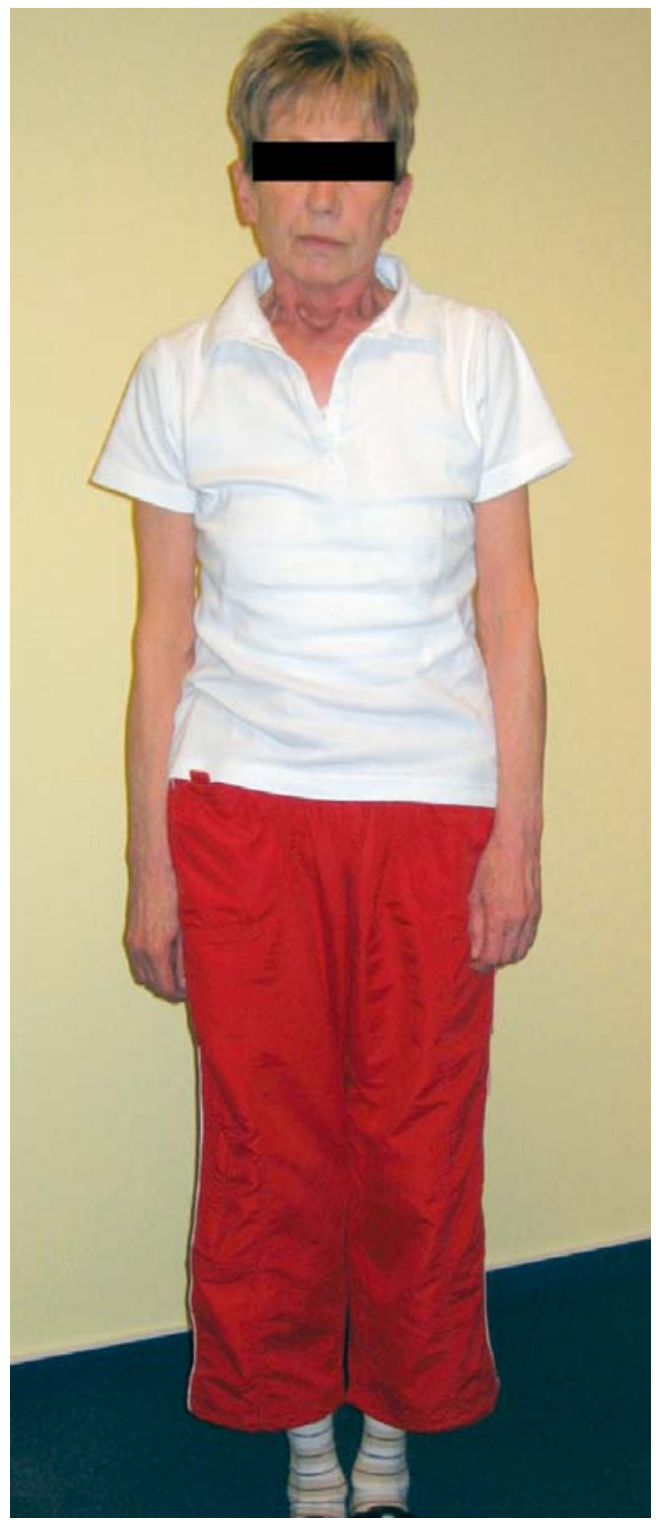

Abb. 1 Patientin mit schwergradiger COPD und einem BMI von $16 \mathrm{~kg} / \mathrm{m}^{2}$.

darüber hinaus eine klare Korrelation der Ausprägung der Mangelernährung mit der Schwere der COPD gezeigt wurde und in denen der Anteil mangelernährter Patienten bei Patientenkollektiven mit mittel- bis sehr schwergradiger COPD bis $38 \%$ reichte [13-17]. Betrachtet man die Gruppe der Patienten mit sehr schwergradiger COPD isoliert, so liegt der Anteil bei nahezu 50\%. Die FettFreie-Masse bzw. der FFM-Index erwiesen sich hinsichtlich der Evaluation des Ernährungszustands auch in anderen Arbeiten, in denen pneumologische Patienten verschiedener Entitäten untersucht wurden, als dem BMI überlegen $[15,18]$.

Die Prävalenz der Adipositas (BMI > $30 \mathrm{~kg} / \mathrm{m}^{2}$ ) beträgt für europäische COPD-Patienten etwa $18 \%$ [19]. Den Daten ist darüber hinaus zu entnehmen, dass der Anteil der Patienten mit Adipositas mit zunehmendem Schwergrad der COPD deutlich abnimmt.

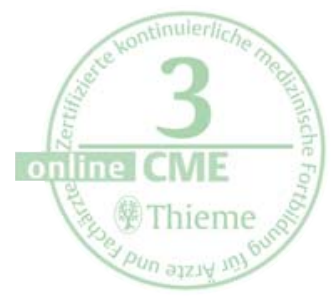


Die isolierte Bestimmung des BMI und die Anwendung der klassischen WHO-Klassifikation sind hinsichtlich der Erfassung einer Mangelernährung bei COPD meist unzureichend. Über die Hälfte der Patienten mit Mangelernährung hat einen BMI > 21 $\mathrm{kg} / \mathrm{m}^{2}$. Das Vorliegen eines BMls $<22 \mathrm{~kg} / \mathrm{m}^{2}$ muss (bei Patienten $>65$ Jahren) meist bereits als Hinweis für das Vorliegen einer Mangelernährung gewertet werden. Über die Messung einer verminderten FFM kann eine eigentliche pulmonale Kachexie nachgewiesen werden. Der Anteil der COPD-Patienten mit Mangelernährung (nutritional depletion) liegt, je nach Schweregrad der respiratorischen Störung, zwischen 25 und 50\%. Der Anteil der COPD-Patienten mit Adipositas beträgt etwa $18 \%$ und nimmt mit zunehmendem Schweregrad der Erkrankung ab.

\section{Welche Relevanz hat der Zustand der Mangelernährung? \\ $\nabla$}

Aus multiplen Studien geht hervor, dass eine Mangelernährung (nutritional depletion) mit einer deutlich erhöhten Mortalität, Morbidität und Hospitalisation bei COPD-Patienten einhergeht und ein gutes Maß für die systemischen Auswirkungen der Erkrankung darstellt $[2,14,18,20,21]$. Wiederholt wurde gezeigt, dass ein verminderter BMI als unabhängiger Risikofaktor einer erhöhten Mortalität zu werten ist. Dies ist in besonderem Maße für Patienten mit schwergradiger COPD zutreffend [14,20-22]. Eine kürzlich veröffentlichte große Metaanalyse $(n=900000$, gemischtes Kollektiv) bestätigte das Vorliegen einer rauchassoziierten respiratorischen Erkrankung als starken Risikofaktor für eine erhöhte Mortalität bei Patienten mit einem BMI < 22,5 [23]. Eine dänische Studie beobachtete ein Kollektiv von COPD-Patienten $(n=2132)$ über einen Zeitraum von 17 Jahren. Hierbei ergab sich ein erhöhtes Mortalitätsrisiko von 1,6 (Männer) bzw. 1,4 (Frauen) bei Vorliegen eines verminderten BMI $\left(<20 \mathrm{~kg} / \mathrm{m}^{2}\right)$ im Vergleich zu normgewichtigen COPD-Patienten [20]. In einem multidimensionalen Graduierungs-Index des COPD-Schweregrades (BODE-Index) wurde, den Ergebnissen verschiedener Studien folgend, ein BMI von $21 \mathrm{~kg} / \mathrm{m}^{2}$ als Wendepunkt einer erhöhten Mortalität vorgeschlagen und evaluiert [4].

Eine verminderte FFM bzw. ein verminderter FFM-Index wurde ebenfalls als unabhängiger Risikofaktor einer erhöhten Mortalität bei COPDPatienten verifiziert. Die Prädiktion ist auch dann zutreffend, wenn der BMI (noch) nicht vermindert ist $[14,16]$. Der Verlust der FFM korreliert zudem invers mit der respiratorischen und peripheren Muskelfunktion [24], der Belastungsfähigkeit [25] und dem allgemeinen Gesundheitszustand (health status) [26].

In einer niederländischen Studie [16] wurde eine Kohorte von 412 COPD-Patienten (GOLD II-IV) hinsichtlich ihres Ernährungszustandes unter-

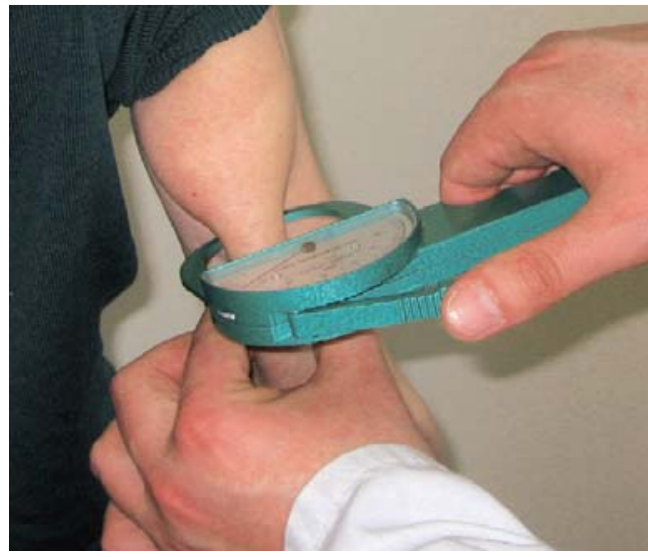

Abb. 2 Messung der Trizepshautfaltendicke mittels Kaliper.

sucht und über 5 Jahre beobachtet. Es fand sich eine signifikante, fast 2-fach erhöhte Mortalität (RR 1,91 bzw. 1,96) bei den Patienten (Anteil 28 \%) mit 1 . vermindertem $\mathrm{BMI}\left(<21 \mathrm{~kg} / \mathrm{m}^{2}\right)$ und vermindertem FFMI $\left(<16 \mathrm{~kg} / \mathrm{m}^{2}\right.$, Männer bzw. $<15$ $\mathrm{kg} / \mathrm{m}^{2}$, Frauen) und 2. bei denen (Anteil 10\%) mit einem $\mathrm{BMI}>21 \mathrm{~kg} / \mathrm{m}^{2}$ und vermindertem FFMI im Vergleich zu den Patienten mit normalem BMI und FFMI. Die FFM wurde als unabhängiger Prädiktor für Mortalität bei COPD bestätigt und erwies sich gegenüber dem BMI als überlegen.

Die Dynamik des Gewichtsverlusts gilt als sensitiver Indikator für die Detektion einer Mangelernährung im Alter [11] und wurde als weiterer unabhängiger Risikofaktor einer erhöhten Mortalität bei COPD-Patienten nachgewiesen. Ein dänisches Kollektiv von 1612 COPD-Patienten (GOLD I-IV) wurde im Abstand von 5 Jahren untersucht und über einen Zeitraum von 14 Jahren beobachtet [27]. Der Anteil der Patienten mit einem Gewichtsverlust von $>1$ BMI-Einheit (etwa $3,8 \mathrm{~kg}$ entsprechend) stieg mit zunehmendem Schweregrad der Erkrankung. Bei den Patienten mit einem FEV1 < 50\% vom Soll betrug der Anteil 35\% (Frauen) bzw. 27\% (Männer). Ab einem Gewichtsverlust von > 1 BMI-Einheit war bei den COPD-Patienten aller Schweregrade ein signifikant erhöhtes Mortalitätsrisiko nachzuweisen. Für einen Gewichtsverlust $>3$ BMI-Einheiten ergab sich ein Mortalitätsrisiko von 1,71 im Vergleich zu Patienten mit stabilem Gewicht.

Die Bewertung des Vorliegens einer Adipositas ist nicht einheitlich. Bei Patienten mit leichter bis mittelgradiger Obstruktion (FEV1 < 50\% vom Soll) zeigt sich, ähnlich der durchschnittlichen Normalbevölkerung, eine angedeutete U-förmige Beziehung zwischen BMI und Sterblichkeit. Während Patienten mit fortgeschrittener COPD (GOLD III und IV) von einer Adipositas prognostisch profitieren, steigt bei leicht- mittelgradiger COPD das Mortalitätsrisiko ab einem $\mathrm{BMI}>30 \mathrm{~kg} / \mathrm{m}^{2}$ tendenziell (statistisch nicht signifikant) wieder an $[20,28]$. Das bedeutet, mit zunehmendem Schweregrad verschiebt sich das BMI-Intervall mit der 
Tab. 2 Folgen der pulmonalen Kachexie.

\section{Folgen der pulmonalen Kachexie}

erhöhte Mortalität

verminderte Belastbarkeit, verminderte Atemkraft erhöhte Infektanfälligkeit

erhöhte Hospitalisationsrate, erhöhte Liegedauer verminderte Lebensqualität

günstigsten Prognose nach oben. Die protektive Wirkung der Adipositas für die Patienten mit schwergradiger COPD wird auch als Obesity Paradox bezeichnet und ist am ehesten Ausdruck eines verminderten kurzfristigen Riskos durch eine verminderte FFM. Bei den frühen Stadien der COPD kommen hingegen, bei eher niedriger COPD-bezogener Mortalität, die langfristig schädigenden Effekte der Adipositas zum Tragen und überwiegen das COPD-bedingte Risiko [29].

Mangelernährung bzw. pulmonale Kachexie ist eine wichtige Komponente der systemischen Auswirkungen der COPD und hat maßgeblichen Einfluss auf die Mortalität und Morbidität der Erkrankung. Ein verminderter BMI, eine verminderte FFM und ein Gewichtsverlust über die Zeit sind unabhängige Prädiktoren einer erhöhten Gesamtmortalität. Adipositas führt bei Patienten mit schwergradiger COPD (GOLD III und IV) zu einem signifikanten Überlebensvorteil.

\section{Welche Konsequenzen ergeben sich aus diesen Erkenntnissen für unsere tägliche Praxis? \\ $\nabla$}

Die Mangelernährung bzw. pulmonale Kachexie ist evident. Rund ein Drittel der COPD-Patienten leiden an dieser systemischen Auswirkung der chronisch obstruktiven Lungenerkrankung. Die Mangelernährung ist Ausdruck komplexer und vielschichtiger Pathomechanismen und geht mit einer Vielzahl verschiedener Phänomene (s. - Tab.2), wie etwa der Schwäche der Atemund Skelettmuskulatur oder einer erhöhten Infektanfälligkeit etc., einher $[6,9,30]$.

Zudem haben COPD-Patienten mit Mangelernährung eine deutlich erhöhte Gesamtmortalität. Das relative Risiko beträgt etwa 1,9 im Vergleich zu normgewichtigen Patienten. Mangelernährte COPD-Patienten bedürfen folglich unserer besonderen Aufmerksamkeit. Die eindeutige Identifikation der Mangelernährung im klinischen Alltag ist nicht ganz einfach. Der Nachweis einer verminderten FFM bedarf z. B. der standardisierten Messung mittels geeigneten BIA-Geräts. Diese Methode steht jedoch nur den wenigsten Kliniken und Praxen zur Verfügung. Wertvolle Hinweise für das Vorliegen einer Mangelernährung ergeben sich jedoch auch über die Erfassung des BMI sowie des Gewichtsverlaufs über die Zeit $[6,10]$. In verschiedenen Studien konnten zumindest kurzfristige Besserungen des Ernährungszustands sowie der assoziierten Prognose mittels ernährungsmedizinischer Interventionen, kombiniert mit körperlicher Aktivität, gesichert werden $[6,10,31]$. Die weitere Optimierung entsprechender Interventionen ist Gegenstand aktueller Untersuchungen. Nicht zuletzt aufgrund des besonderen Studiendesigns und der nötigen Kontrolle vielfältiger Einflussfaktoren gestalten sich entsprechende Studien jedoch schwierig.

Das Problem der Mangelernährung bzw. pulmonalen Kachexie bei COPD ist evident; ca. ein Drittel der Patienten sind betroffen. Das Morbiditäts- und Mortalitätsrisiko ist hierbei signifikant erhöht. Mit zunehmendem Schweregrad der Erkrankung steigt die Relevanz des Problems. Die ernährungsmedizinische Intervention der Mangelernährung beinhaltet das Erkennen einer Depletion und das Einleiten gegenregulatorischer Maßnahmen (Ernährung und körperliches Training). Die Erfassung des Gewichtsverlaufes und die Ermittlung des BMI müssen zu den Basisparametern der regelmäßigen Untersuchung von COPD-Patienten gehören.

\section{Literatur}

1 Buist AS, McBurnie MA, Vollmer WM et al. International Variation in the Prevalence of COPD (The BOLD Study): a Population-based Prevalence Study. Lancet 2007; 370: $741-750$

2 Agusti AGN, Noguera A, Sauleda J et al. Systemic effects of chronic obstructive pulmonary disease. Eur Respir J 2003; 21: 347-360

3 MacNee W. Update in Chronic Obstructive Pulmonary Disease 2007. Am J Respir Crit Care Med 2008; 177: 820 $-829$

4 Celli BR, Cote CG, Marin JM et al. The Body-Mass Index, Airflow Obstruction, Dyspnea, and Exercise Capacity Index in Chronic Obstructive Pulmonary Disease. N Engl J Med 2004; 350: 1005-1012

5 Bösch D, Feierabend M, Becker A. Ambulante COPD-Patientenschulung (ATEM) und BODE-Index. Pneumologie 2007; 61: 629-635

6 Bösch D. COPD - ernährungsmedizinische Betreuung. In: Schauder P, Ollenschläger G (Hrsg). Ernährungsmedizin - Prävention und Therapie, 3. Auflage. München: Elsevier; 2006: $1001-1007$

7 Calverley PMA, Anderson JA, Celli B et al. Salmeterol and Fluticasone Propionate and Survival in Chronic Obstructive Pulmonary Disease. N Engl ] Med 2007; 356: 775-789

8 Tashkin DP, Celli B, Senn S et al. A 4-Year Trial of Tiotropium in Chronic Obstructive Pulmonary Disease. N Engl ] Med 2008: 359: $1543-1554$

9 Statistisches Bundesamt. Leben in Deutschland. Ergebnisse des Mikrozensus 2005.

10 Bauer JM, Wirth R, Volkert D et al. Malnutrition, Sarkopenie und Kachexie im Alter - Von der Pathophysiologie zur Therapie. Ergebnisse eines internationalen Expertenmeetings der BANSS-Stiftung. Dtsch Med Wochenschr 2008; 133: 305-310

11 Volkert D, Schlierf G. Ernährung im Alter. In: Schauder P, Ollenschläger G, HrsgErnährungsmedizin - Prävention und Therapie, 3. Auflage. München: Elsevier; 2006: 367 $-374$ 
12 Vermeeren MAP, Creutzberg EC, Schols AMW] et al. Prevalence of nutritional depletion in a large out-patient population of patients with COPD. Respir Med 2006; 100 : $1349-1355$

13 Schols AM, Soeters PB, Dingemans AM et al. Prevalence and characteristics of nutritional depletion in patients with stable COPD eligible for pulmonary rehabilitation. Am Rev Respir Dis 1993; 147: 1151 - 1156

14 Vestbo J, Prescott E, Almdal T et al. Body Mass, Fat-Free Body Mass, and Prognosis in Patients with Chronic Obstructive Pulmonary Disease from a Random Population Sample. Findings from the Copenhagen City Heart Study. Am J Respir Crit Care Med 2006; 173: 79-83

15 Budweiser S, Meyer K, Jörres RA et al. Nutritional depletion and its relationship to respiratory impairment in patients with chronic respiratory failure due to COPD or restrictive thoracic diseases. Eur J Clin Nutr 2008; 62: 436 - 443

16 Schols AMWJ, Broekhuizen R, Weling-Scheepers CA, Wouters EF. Body Composition and Mortality in Chronic Obstructive Pulmonary Disease. Am J Clinical Nutrition 2005; 82: 53-59

17 Ischaki E, Papatheodorou G, Gaki E. Body Mass and FatFree Mass Indices in COPD -Relation with Variables Expressing Disease Severity. Chest 2007; 132: 164- 169

18 Cano1 NJM, Roth H, Court-Fortuné l et al. Nutritional depletion in patients on long-term oxygen therapy and/or home mechanical ventilation. Eur Respir 」 2002; 20: $30-37$

19 Steuten LM, Creutzberg EC, Vrijhoef HJ et al. COPD as a Multicomponent Disease: Inventory of Dyspnoea, Underweight, Obesity and Fat Free Mass Depletion in Primary Care. Prim Care Respir ] 2006; 15: 84-91

20 Landbo C, Prescott E, Lange $P$ et al. Prognostic Value of Nutritional Status in Chronic Obstructive Pulmonary Disease. Am J Respir Crit Care Med 1999; 160: 1856 1861

21 Chailleux E, Laaban JP, Veale D. Prognostic Value of Nutritional Depletion in Patients with COPD Treated by Longterm Oxygen Therapy. Chest 2003; 123: 1460 - 1466
22 Schols AM, Slangen J, Volovics L, Wouters EF. Weight Loss is a Reversible Factor in the Prognosis of Chronic Obstructive Pulmonary Disease. Am J Respir Crit Care Med 1998; 157: $1791-1797$

23 Prospective Studies Collaboration. Body-mass index and cause-specific mortality in 900000 adults: collaborative analyses of 57 prospective studies. Lancet 2009; 373: 1083-1096

24 Engelen MP, Schols AM, Baken WC et al. Nutritional Depletion in Relation to Respiratory and Peripheral Skeletal Muscle Function in Out-Patients with COPD. Eur Respir 1994; 7: $1793-1797$

25 Schols AM, Soeters PB, Dingemans AM et al. Prevalence and Characteristics of Nutritional Depletion in Patients with Stable COPD Eligible for Pulmonary Rehabilitation. Am Rev Respir Dis 1993; 147: 1151-1156

26 Mostert R, Goris A, Weling-Scheepers C et al. Tissue Depletion and Health Related Quality of Life in Patients with Chronic Obstructive Pulmonary Disease. Respir Med 2000; 94: 859-867

27 Prescott E, Almdal T, Mikkelsen KL et al. Prognostic Value of Weight Change in Chronic Obstructive Pulmonary Disease: Results from the Copenhagen City Heart Study. Eur Respir J 2002; 20: 539- 544

28 Jee SH, Sull JW, Park J et al. Body-Mass Index and Mortality in Korean Men and Women. N Engl J Med 2006; 355: $779-787$

29 Franssen FME, O'Donnell DE, Goossens GH et al. Obesity and the lung: 5 - Obesity and COPD. Thorax 2008; 63: $1110-1117$

30 Agusti A. Systemic Effects of Chronic Obstructive Pulmonary Disease - What We Know and What We Don't Know (but Should). Proc Am Thorac Soc 2007; 4: 522 525

31 King DA, Cordova F, Scharf SM. Nutritional Aspects of Chronic Obstructive Pulmonary Disease. Proc Am Thorac Soc 2008; 5: 519-523

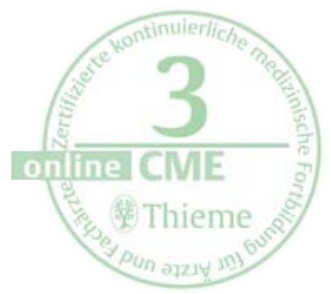




\section{CME-Fragen Ernährungszustand und Körperzusammensetzung bei COPD}

1 Welche Aussage hinsichtlich der Bestimmung

des Body-Mass-Indexes (BMI) ist richtig?

A Über die Bestimmung des BMI lässt sich eine Mangelernährung eindeutig bestimmen.

B Mittels BMI kann die Körperzusammensetzung im Sinne eines Zweikompartment-Modells erfasst werden.

C Ein BMI $<22 \mathrm{~kg} / \mathrm{m}^{2}$ ist (bei älteren Patienten) bereits ein Hinweis für das mögliche Vorliegen einer Mangelernährung.

D Definitionsgemäß liegt bei einem COPD-Patienten eine Mangelernährung erst dann vor, wenn der BMI $<18,5 \mathrm{~kg} / \mathrm{m}^{2}$ ist.

E Der BMI wird mittels Bioimpedanzanalyse ermittelt.

2 Welche Aussage zur Fett-Freien-Masse (FFM) ist im Hinblick auf eine pulmonale Kachexie nicht richtig?

A Sie hat im Vergleich zum BMI eine deutlich höhere Aussagekraft.

B Sie ist ein indirektes Maß der Muskelmasse.

C Eine weitere Differenzierung in Zellmasse und extrazelluläre Masse ist möglich.

D Sie kann auch bei normalem BMI eine pulmonale Kachexie nachweisen.

E Eine Verminderung geht mit einem verbesserten Überleben einher.

3 Welche Aussage bzgl. des Ernährungszustandes bei COPDPatienten ist nicht richtig?

A Der Anteil mangelernährter Patienten beträgt im Mittel ca. $30 \%$.

B Der Anteil mangelernährter Patienten steigt mit zunehmendem respiratorischen Schweregrad der Erkrankung von ca. 25 auf fast $50 \%$.

C Der Anteil adipöser Patienten beträgt im Mittel ca. $18 \%$.

D Der Anteil adipöser Patienten steigt mit zunehmendem respiratorischen Schweregrad der Erkrankung.

E Der Ernährungszustand wird nicht nur über die Bestimmung des Körpergewichts definiert.

4 Welches ist keine typische Folge einer Mangelernährung? A eine erhöhte Mortalität

B eine erhöhte Hospitalisationsrate

C eine erhöhte Infektanfälligkeit

D eine bessere Lungenfunktion

E eine verminderte Lebensqualität
5 Welches ist kein unabhängiger Prädiktor einer erhöhten

Mortalität bei Vorliegen einer schwergradigen COPD?

A eine verminderte Fett-Freie-Masse

B ein BMI von $20 \mathrm{~kg} / \mathrm{m}^{2}$

C ein BMI von $18 \mathrm{~kg} / \mathrm{m}^{2}$

D ein ungewollter Gewichtsverlust von $5 \mathrm{~kg}$ im letzten halben Jahr

E eine Adipositas mit einem BMI von $31 \mathrm{~kg} / \mathrm{m}^{2}$

6 Welche Aussage ist richtig?

A Der BMI und der Gewichtsverlauf sollte bei COPDPatienten lediglich bei Vorliegen gastrointestinaler Beschwerden ermittelt werden.

B Die Messung der Fett-Freien-Masse ist simpel und bei jeder Aufnahme eines COPD-Patienten durchzuführen.

C Die Relevanz einer Mangelernährung betrifft lediglich Patienten mit einer schwergradigen COPD und einem $\mathrm{BMI}<18,5 \mathrm{~kg} / \mathrm{m}^{2}$.

D Patienten mit leichtgradiger COPD profitieren prognostisch von einer Adipositas Grad 2.

E Ein COPD-Patient mit einem BMI von $25 \mathrm{~kg} / \mathrm{m}^{2}$ sollte sein Gewicht möglichst halten.

7 Welche Aussage ist falsch?

A Die regelmäßige Erfassung von BMI und Gewichtsverlauf sind sinnvolle Untersuchungen bei jedem COPD-Patienten.

B Bei Hinweisen für das Vorliegen einer Mangelernährung wäre die Bestimmung der Fett-Freien-Masse wünschenswert.

C Nach Erkennen einer pulmonalen Kachexie sollten gegenregulatorische Maßnahmen eingeleitet werden.

D Sportliche Betätigung sollte bei Nachweis einer pulmonalen Kachexie möglichst gemieden werden.

E Das Problem der pulmonalen Kachexie betrifft auch Patienten anderer respiratorischer Entitäten.

8 Was wird auch als obesity paradox bezeichnet?

A Die erhöhte Mortalität bei Patienten mit Adipositas und COPD.

B Die protektive Wirkung der Adipositas bei Patienten mit schwergradiger COPD.

C Die deutliche Besserung der Lungenfunktion mit zunehmender Adipositas.

D Die deutliche Verschlechterung der Lungenfunktion mit zunehmender Adipositas.

E Die deutliche Gewichtszunahme bei Abnahme der Muskelmasse. 
9 Welche Aussage trifft für einen 66-jährigen COPD-

Patienten mit einer FEV1 von $40 \%$ vom Soll, einem BMI von $20 \mathrm{~kg} / \mathrm{m}^{2}$, einem FFMI von $14,5 \mathrm{~kg} / \mathrm{m}^{2}$ und einem Gewichtsverlust von $4 \mathrm{~kg}$ in den letzten 3 Monaten nicht zu?

A Der BMI liegt laut WHO-Klassifikation im Normbereich.

B Es liegen ein normaler Ernährungszustand und eine normale Körperzusammensetzung vor.

C Es liegt eine pulmonale Kachexie bzw. eine Mangelernährung mit Verminderung der Muskelmasse vor.

D Der Patient hat ein signifikant erhöhtes Mortalitätsrisiko.

E Der Patient sollte ernährungsmedizinisch betreut werden.
10 Welche Aussage trifft für einen 66-jährigen COPD-

Patienten mit einer FEV1 von $\mathbf{4 0} \%$ vom Soll, einem BMI von $32 \mathrm{~kg} / \mathrm{m}^{2}$, einem FFMI von $17 \mathrm{~kg} / \mathrm{m}^{2}$ und einem seit 6 Monaten stabilen Gewicht zu?

A Der Patient ist adipös und sollte lieber keine sportliche Aktivität aufnehmen.

B Der Patient ist adipös und sollte sein Gewicht um ca. $10 \mathrm{~kg}$ reduzieren.

C Der Patient ist adipös und hat keinen Hinweis auf eine verminderte Muskelmasse.

D Der Patient hat ein signifikant erhöhtes Mortalitätsrisiko.

E Der Patient braucht hinsichtlich seines Gewichtes in Zukunft nicht mehr untersucht zu werden.

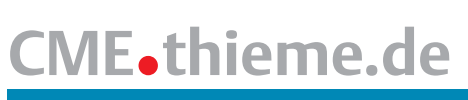

\section{CME-Teilnahme}

- Viel Erfolg bei lhrer CME-Teilnahme unter http://cme.thieme.de

- Diese Fortbildungseinheit ist 12 Monate online für eine CME-Teilnahme verfügbar.

- Sollten Sie Fragen zur Online-Teilnahme haben, unter http://cme.thieme.de/hilfe finden Sie eine ausführliche Anleitung. 